\title{
An Adult Patient with a Rare Subform of Recessive Dystrophic Epidermolysis Bullosa Inversa (Gedde-Dahl)
}

\author{
Tina Giner ${ }^{1}$, Cristina $\operatorname{Has}^{2}$, Matthias Goebeler ${ }^{3}$, Martin Leverkus ${ }^{4}$, Eva-Bettina Bröcker ${ }^{1}$ and \\ Henning Hamm ${ }^{*}, 1$ \\ ${ }^{I}$ Department of Dermatology, University Clinics of Würzburg, Josef-Schneider-Str. 2, D-97080 Würzburg, Germany \\ ${ }^{2}$ Department of Dermatology, University Clinics of Freiburg, Hauptstr. 7, D-79104 Freiburg, Germany \\ ${ }^{3}$ Department of Dermatology, University Clinics of Giessen, Gaffkystr. 14, D-35385 Giessen, Germany \\ ${ }^{4}$ Department of Dermatology, University Clinics of Magdeburg, Leipziger Str. 44, D-39120 Magdeburg, Germany
}

\begin{abstract}
A 53-year-old male patient was referred for evaluation of recurrent erosions of inguinal and anogenital areas, both lower legs and the oral mucosa. In addition, almost all nails and several teeth were lacking, and the residual teeth showed enamel defects. History revealed congenital absence of skin of large parts of the lower legs and widespread blistering in early childhood. Antigen mapping showed immunoreactivity against collagen XVII, laminin 332, collagen IV and collagen VII at the epidermal side of a skin split. Molecular analysis resulted in the identification of compound heterozygous mutations in exon $3(\mathrm{c} .425 \mathrm{~A}>\mathrm{G})$ and exon 74 (p.Arg2069Cys) of the COL7A1 gene which has been reported in one family before. We conclude that our patient suffers from recessive dystrophic epidermolysis bullosa (EB) inversa, a rare subtype of dystrophic EB first described by Gedde-Dahl. Remarkable is the relatively mild course with clinical features formerly known as Bart syndrome.
\end{abstract}

Keywords: Dystrophic epidermolysis bullosa, collagen type VII.

Dystrophic epidermolysis bullosa (EB) is a heterogeneous genetic disease characterized by skin fragility and blister formation. Modes of inheritance of dystrophic EB are either autosomal dominant (approximately 20\%) or autosomal recessive (approximately 80\%) [1, 2]. More than 400 different mutations of the COL7A1 gene encoding type VII collagen (Human Gene Mutation Database, http://www.hgmd.cf.ac.uk/ac/index.php), the major structural component of anchoring fibrils at the dermalepidermal junction, are on record [3, 4].

A 53-year-old Caucasian male presented with chronic circumscribed blistering of the inguinal folds, anogenital area, extensor surfaces of the lower legs and the oral mucosa including the tongue. He reported to have spent the first several months of life in hospital because of congenital absence of skin on the anterior parts of the lower legs and feet which subsequently healed without scarring. During infancy and early childhood he had suffered from extensive blistering of his body including the palms and developed subtotal loss of toe- and fingernails later in life. Adult-onset diabetes mellitus with documented microangiopathy had led to the amputation of both great toes. The patient denied any blistering or nail defects in family members.

Clinical examination showed inguinal erosions and a small blister in the right inguinal fold. In addition, he

\footnotetext{
*Address correspondence to this author at the Department of Dermatology, University Clinics of Würzburg, Josef-Schneider-Str. 2, D-97080 Würzburg, Germany; Tel: 49-931-201-26738; Fax: 49-931-201-26462;

E-mail: hamm_h@klinik.uni-wuerzburg.de
}

suffered from erythema of the perianal area and the right side of the scrotum with several erosions up to $5 \mathrm{~cm}$ in size as well as leukoplakia of the right margin of the tongue. On the lower legs he had two superficial ulcers and rarefaction of hairs. Apart from nail residues on 2 fingers all nails were absent. Several teeth were lacking, others had enamel defects.

A skin biopsy from the right groin revealed subepidermal blistering, consistent with the diagnosis of junctional or dystrophic EB. Of note, unaffected skin was not extraordinarily vulnerable and we could not induce visible blistering within 3 hours after heavily rubbing the skin. Antigen mapping of a biopsy of clinically unaffected skin (left forearm) showed immunoreactivity against collagen XVII, laminin 332, collagen IV and collagen VII in the roof of a minimal skin split, corresponding to dystrophic EB.

To gain deeper insight of the underlying genetic defect, molecular genetic analysis from genomic DNA was performed. All exons and exon-intron boundaries of the COL7A1 gene were analysed by polymerase chain reaction (PCR) followed by sequencing [3]. Interestingly, we found heterozygous mutations in the COL7A1 gene in exon 3 (c.425A $>\mathrm{G}$ ) and exon 74 (p.Arg2069Cys). Thus, a diagnosis of autosomal recessive dystrophic EB inversa was made. The mutation c. $425 \mathrm{~A}>\mathrm{G}$ in exon 3 has been described to lead to abnormal splicing [5], the other mutation, c. $6205 \mathrm{C}>\mathrm{T}$ in exon 74, causes replacement of codon 2069 for arginine by a codon for cysteine. The same molecular constellation of compound heterozygosity has been found in one family before with similar clinical features [6]. 

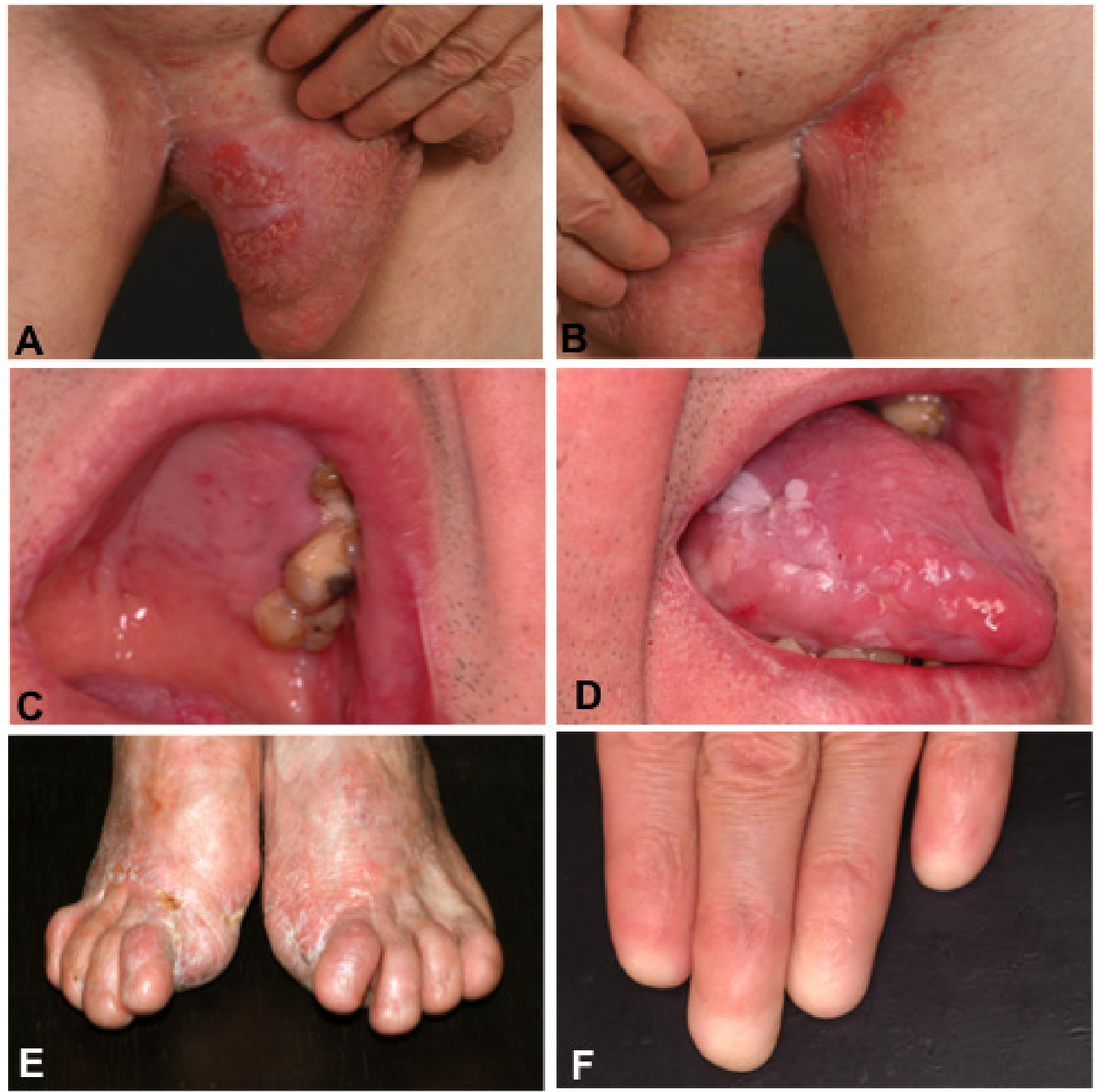

Fig. (1). A, B) Groins and scrotal region of the patient showing erythema and erosions up to $5 \mathrm{~cm}$ in diameter with fibrinous coating. C) Oral cavity showing a lack of several teeth and enamel defects in the remaining ones, as well as mild erosions on the hard palate. D) Leukoplakia of the right margin of the tongue. E) Feet with missing or grossly dystrophic toenails. Note amputated first toes due to adult-onset diabetes mellitus. F) Loss of fingernails.

Symptomatic treatment of intertriginous lesions included local application of $0.1 \%$ aqueous crystal violet solution, soft zinc paste and insertion of non-adhesive compresses which improved the patient's symptoms. Oral treatment was started with panthenol lozenges and antiseptic fluids.

Dystrophic epidermolysis bullosa and allelic blistering disorders with mutations in the type VII collagen gene can be divided into various forms. Dominant dystrophic EB occurs in a mild (Cockayne-Touraine) and severe (Pasini) clinical subtype. Recessive forms of dystrophic EB present in a severe generalized form (EB Hallopeau-Siemens) and in less pronounced forms (EB non-Hallopeau-Siemens), further classified into mitis, localized and inverse subtypes with a broad range of clinical severity. Related conditions with sublamina densa split formation have been described as Bart syndrome, bullous dermolysis of the newborn, and as pretibial and pruriginous forms of EB [1,2].

The subtype of dystrophic EB present in our patient was first described by Gedde-Dahl in 1971 as recessive dystrophic EB inversa [7]. Until today, only a few cases of 
this subtype have been reported [6, 8]. While the overall number of reported cases with recessive dystrophic EB inversa is lower than 20, only two patients with the identical underlying mutation have been published so far [6]. Skin and mucosal involvement typically improve over the years, with generalized blistering compromising sufferers during early childhood and confinement of lesions to flexures and the oral mucosa in adult life. This relatively mild course in later years could also be noticed in our patient. Until genetic evidence for heterogeneity, the present clinical triad of congenital localized absence of skin, blistering of skin and mucous membranes and severe nail dystrophy was referred to as Bart syndrome [9]. We and others [2, 10] propose not to use this term anymore, as it has become clear that "Bart syndrome" subsumes several different underlying genetic defects which can be better defined as various subforms of epidermolysis bullosa.

\section{REFERENCES}

[1] Varki R, Sadowski S, Uitto J, et al. Epidermolysis bullosa. II. Type VII collagen mutations and phenotype-genotype correlations in the dystrophic subtypes. J Med Genet 2007; 44: 181-92.

[2] Fine J-D, Eady RAJ, Bauer EA, et al. The classification of inherited epidermolysis bullosa (EB): Report of the Third International Consensus Meeting on Diagnosis and Classification of EB. J Am Acad Dermatol 2008; 58: 931-50.
[3] Kern JS, Kohlhase J, Bruckner-Tuderman L, et al. Expanding the COL7A1 mutation database: novel and recurrent mutations and unusual genotype-phenotype constellations in 41 patients with dystrophic epidermolysis bullosa. J Invest Dermatol 2006; 126: 1006-12.

[4] van den Akker PC, van Essen AJ, Kraak MM, et al. Long-term follow-up of patients with recessive dystrophic epidermolysis bullosa in the Netherlands: expansion of the mutation database and unusual phenotype-genotype correlations. J Dermatol Sci 2009; 56: 9-18.

[5] Gardella R, Belletti L, Zoppi N, et al. Identification of two splicing mutations in the collagen type VII gene (COL7A1) of a patient affected by the localisata variant of recessive dystrophic epidermolysis bullosa. Am J Hum Genet 1996; 59: 292-300.

[6] Kahofer P, Bruckner-Tuderman L, Metze D, et al. Dystrophic epidermolysis bullosa inversa with COL7A1 mutations and absence of GDA-J/F3 protein. Pediatr Dermatol 2003; 20: 243-8.

[7] Gedde-Dahl T Jr. Epidermolysis bullosa. A clinical, genetic and epidemiological study. Baltimore, MD: John Hopkins University Press 1971.

[8] Medenica L, Lens M. Recessive dystrophic epidermolysis bullosa: presentation of two forms. Dermatol Online J 2008 15; 14: 2 .

[9] Bart BJ, Gorlin RJ, Anderson E, Lynch FW. Congenital localized absence of skin and associated abnormalities resembling epidermolysis bullosa. A new syndrome. Arch Dermatol 1966; 93: 296-304.

[10] Gutzmer R, Herbst RA, Becker J, et al. Bart syndrome - separate entity or a variant of epidermolysis bullosa? Hautarzt 1997; 48: 640-4. 